\title{
Diabetic cardiomyopathy: a review of literature on clinical status and meta-analysis of tissue doppler diagnostic method and the clinical value of intensive glycemic control
}

\author{
Aref Albakri* \\ St-Marien hospital Bonn Venusberg, Department of Internal Medicine, Bonn, Germany
}

\begin{abstract}
Abbreviations: ACC: American College of Cardiology; ACE: Angiotensin Converting Enzyme; AGE: Advance Glycation Endproducts; AHA: American Heart Association; ANP: Atrial Natriuretic Peptide; ARB: Angiotensin Receptor Blocker; ATLAS: Assessment of Treatment with Lisinopril and Survival; BNP: Brain Natriuretic Peptide; CAD: Coronary Artery Disease; CHF: Congestive Heart Failure; CHS: Cardiovascular Health Study; CMRI: Cardiac Magnetic Resonance Imaging; CPC: Cardiac Progenitor Cells; CRP: C - Reactive Protein; DM: Diabetes Mellitus; ESC: European Society of Cardiologists; FFA: Free Fatty Acid; Hba: Glycated Hemoglobin; HF: Heart Failure; HT: Hypertension; IL-6: Interleukin-6; LVEF: Left Ventricular Ejection Fraction; MESA: Multi-Ethnic Study of Atherosclerosis; MFR: Myocardial Flow Reserve; MI: Myocardial Infarction; MPI: Myocardial Perfusion Imaging; MRA: Mineralocorticoid Receptor Antagonist; RAAS: Renin-Angiotensin-Aldosterone System; RESOLVD: Randomized Evaluation of Strategies for Left Ventricular Dysfunction; SHS: Strong Heart Study; SOLVD: Studies of Left Ventricular Dysfunction; T1DM: Type 1 Diabetes Mellitus; T2DM: Type 2 Diabetes Mellitus; UKPDS: United Kingdom Prospective Diabetes Study.
\end{abstract}

\section{Introduction}

The prevalence of diabetes mellitus (DM) is approaching pandemic proportions. Current estimates project DM will affect 300 million people worldwide by 2025 [1]. DM predisposes the affected individuals to serious cardiovascular complications, implicated as the leading cause of diabetic-associated morbidity and mortality [2]. Thus, there is need for a precise clinical understanding of the exact role of DM in the pathophysiology of structural and functional cardiac abnormalities to inform prompt and appropriate therapeutic interventions. To advance such an understanding, this review aggregates available experimental and clinical knowledge on the definition, epidemiology, risk factors, pathophysiology, diagnosis and clinical management of diabetic cardiomyopathy.

\section{Description}

Historical Context:The historical connection between diabetes mellitus and heart failure has been well documented in literature, but the recognition of diabetic cardiomyopathy as a distinct clinical entity has remained a subject of intense scholarly discussion. In 1881, Leyden et al. [3] made the seminal description of diabetic-associated heart disease captured in the statement, "heart failure is a frequent and noteworthy complication of diabetes mellitus". In 1888, Mayer [4] further described an association between diabetic-associated heart disease and abnormalities in metabolism. Later in 1954, Lundbaek [5] observed myocardial dysfunction was a frequent DM-related complication in about two thirds of elderly DM patients. In 1969, Lundbaek [6] again made the initial suggestion for a specific diagnosis of DM-related myocardial dysfunction. While these early studies would have anchored the understanding of diabetic cardiomyopathy as a clinical entity, the publications were in non-English journals and translational research issues undermined both scholarly and clinical understanding as well as consensus.

In 1972, a landmark study by Rubler et al. [7], currently embraced as a citation classic, produced the most compelling evidence of a cardiomyopathy-like condition resulting directly from DM rather than indirectly from concomitant structural heart diseases. The study assessed post-mortem findings of four patients with DM-related nephropathy and heart failure (HF) in the absence of any demonstrable valvular, congenital or hypertensive heart disease, alcoholism or coronary artery disease (CAD). Rubler et al. [7] concluded that the four patients suffered from a novel DM-related myocardial dysfunction, and thus originated and popularized the term "diabetic cardiomyopathy". The term was based on the definition of cardiomyopathy: a cardiac condition characterized by structural and/or functional myocardial abnormalities in the absence of any demonstrable CAD, hypertension (HT), valvular or congenital heart disorders [8]. Table 1 provides a summary of documented historical understanding of diabetic cardiomyopathy.

Clinical Definition: About five decades since Rubler et al. [7] landmark study, diabetic cardiomyopathy has remained the subject of much research and controversy. The focus of current research emphasis is also skewed towards obtaining the best clinical management strategies rather and less on epidemiology and pathophysiology. Rubler et al. [7] originally defined diabetic cardiomyopathy as ventricular dysfunction in the absence of CAD and HT. To date, diabetic cardiomyopathy lacks a standardized or a strict clinical definition, with authors providing various definitions (Table 2). The various definitions identify diabetic cardiomyopathy generally as a myocardial dysfunction in the absence of underlying structural heart disease and/or hypertension. Litwin

*Correspondence to: Aref Albakri, St-Marien hospital Bonn Venusberg, Department of Internal Medicine, Bonn, Germany, E-mail: arefalbakri@yahoo.com

Key words: diabetic cardiomyopathy, glycemic control, tissue doppler, diabetes mellitus

Received: September 06, 2018; Accepted: September 15, 2018; Published: September 172018 
Albakri A (2018) Diabetic cardiomyopathy: a review of literature on clinical status and meta-analysis of tissue doppler diagnostic method and the clinical value of intensive glycemic control

\begin{tabular}{l} 
Table 1. Historical Context of Diabetic Cardiomyopathy \\
\begin{tabular}{|l|l|l|}
\hline Year & First Author [Ref \#] & Description \\
\hline 1881 & Leyden et al. [3] & $\begin{array}{l}\text { Provided the initial description of a heart disease } \\
\text { associated with diabetes mellitus. }\end{array}$ \\
\hline 1888 & Mayer [4] & $\begin{array}{l}\text { Described an association between diabetic-associated } \\
\text { heart disease and metabolic abnormalities. }\end{array}$ \\
\hline 1954 & Lundbaek [5] & $\begin{array}{l}\text { Described myocardial dysfunction as a common } \\
\text { complication in elderly DM patients. }\end{array}$ \\
\hline 1969 & Lundbaek [6] & $\begin{array}{l}\text { Suggested the need for developing specific diagnosis } \\
\text { criteria for myocardial dysfunction associated with } \\
\text { diabetes mellitus. }\end{array}$ \\
\hline 1972 & Rubler et al. [7] & $\begin{array}{l}\text { Originated the term diabetic cardiomyopathy, describing } \\
\text { the condition as myocardial dysfunction in the absence of } \\
\text { coronary artery disease, hypertension and alcohol. }\end{array}$ \\
\hline
\end{tabular} \\
\hline
\end{tabular}

Table 2. Summary of Current Definitions of Diabetic Cardiomyopathy

\begin{tabular}{|l|l|}
\hline First Author [Ref \#] & Definition \\
\hline Asghar et al. [9] & $\begin{array}{l}\text { "A primary disease process developing secondary of metabolic } \\
\text { insult, resulting in structural and functional abnormalities of the } \\
\text { myocardium leading to heart failure" }\end{array}$ \\
\hline Mardikar et al. [10] & $\begin{array}{l}\text { "Ventricular dysfunction (both systolic and diastolic) occurring } \\
\text { in the absence of coronary artery disease and hypertension" }\end{array}$ \\
\hline Mehta et al. [11] & $\begin{array}{l}\text { "The presence of myocardial dysfunction in the absence of } \\
\text { coronary artery disease and hypertension" }\end{array}$ \\
\hline Westermeier et al. [12] & $\begin{array}{l}\text { "A disease characterized by morphological, functional and } \\
\text { metabolic changes in the heart produced by Type 2 DM } \\
\text { (T2DM)" }\end{array}$ \\
\hline $\begin{array}{l}\text { Bell [13]/Fein et al. } \\
{[14]}\end{array}$ & $\begin{array}{l}\text { "Ventricular dysfunction in diabetic patients that is out of } \\
\text { proportion to their underlying vascular disease" }\end{array}$ \\
\hline
\end{tabular}

[15] states it is clinically significant to have a generalized definition because a strict definition of changes in cardiac structure secondary to DM and resulting in myocardial dysfunction would mean diabetic cardiomyopathy does not exist in patients with Type 1 diabetes mellitus (T1DM). However, the current definitions of diabetic cardiomyopathy excludes cardiomyopathy secondary to structural heart diseases, which exists in diabetic patients but poses a clinical challenge because of the difficulty in assessing the contribution of diabetic cardiomyopathy to the overall ventricular dysfunction [16].

Epidemiology: Over the past three decades, epidemiological link between DM and the development of heart failure in the absence of any demonstrable structural or atherosclerotic cardiovascular disease has been well demonstrated in both large-scale population-based and cross-sectional studies.

Large-Scale Studies: The reported prevalence of HF in DM patients is significantly higher than that reported in the general population. The prevalence in the general population is 1 to $4 \%$ while in diabetic population is $12 \%$ [17], which increases considerably to $22 \%$ in DM patients aged $>64$ years [18]. Further, up to three thirds of all admitted HF patients have been diagnosed with DM [19] and $30 \%$ of inpatients with cardiac failure [20], which rises to four times for those newly diagnosed with HF [21]. The Framingham Heart Study [22] found 2.4 and 5.1 times increase in incidence of HF in diabetic men and women respectively in comparison to age-matched cohort adjusted for hypertension, obesity, CAD and dyslipidemia. Breton et al [23] nationwide study yielded similar findings. Several other largescale population-based studies have supported these findings. The Cardiovascular Health Study (CHS) [24] recruiting patients $>65$ years associated DM with increased HF incident. The Strong Heart Study (SHS) [25] reported association between DM and increased LV mass, wall thickness and systolic dysfunction in comparison to age-matched cohort. The Multi-Ethnic Study of Atherosclerosis (MESA) study reported inter-racial differences in LV mass, volume and function among DM patients [26].

Cross-Sectional Studies: Cross-sectional studies also support the findings of large-scale studies reporting a significantly higher incidence of HF among DM patients [18]. Nichols et al. [27] reported 12\% T2DM patients with $\mathrm{HF}$ at presentation with an annual incidence of $3.3 \%$. In a related study of over three years, Aronow et al. [28] report the incidence of HF in DM patients was significantly higher (39\%) compared to nonDM patients (23\%) and having a relative risk of 1.3 to develop HF. The United Kingdom Prospective Diabetes Study (UKPDS) [29] reports higher prevalence of HF in T2DM patients with elevated levels of glycated hemoglobin ( $\mathrm{Hba}$ ). The study further revealed that for every $1 \%$ rise in $\mathrm{Hba}$, there is a corresponding $8 \%$ increase in the likelihood of developing HF. Cheung et al. [30] and sub-group analysis of the MESA study [31] support the association between retinal arteriolar narrowing and LV remodeling increasing the risk of HF in DM patients with retinopathy. Both largescale and cross-sectional studies support the over-representation of DM patients in large population-based HF trials including Studies of Left Ventricular Dysfunction (SOLVD: 26\%) [32]; Assessment of Treatment with Lisinopril and Survival (ATLAS: 19\%) [33]; and Randomized Evaluation of Strategies for Left Ventricular Dysfunction (RESOLVD: 27\%) [34].

Risk factors: The two main factors that have been implicated to predispose diabetic patients to increased risk of developing DMassociated HF are (a) hyperglycemia and (b) hypertension [9]. However, these two factors lack conclusive research support for their association with increased risk of DM-associated HF, and thus warrants the need for additional research to elucidate the association.

Hyperglycemia: Current research findings on whether hyperglycemia elevates the risk of developing HF in patients with diabetic cardiomyopathy remains fragmented, and at most, inconclusive. Although the large scale UKPDS trial [29] associated poor glycemic control with increased risk of developing HF, on the other hand, intensive glycemic control did not convey any significant acute effect on reducing the incident of HF. However, in a recent study of T2DM patients, Andersen et al. [35] observed improved rate of systolic strain and decreased LV mass associated with improved Hba fructosamine levels, which was independent of changes in blood pressure lowering effects (Figure 1). There was also an improvement in diastolic function in the first three months, which reversed to normal in 12months. In a related study on the determinants of subclinical diabetic heart disease, Fang et al. [36] reported similar findings of the association between lower $\mathrm{Hba}$ and improved systolic strain. In a sub-group analysis of the UKPDS trial followed for 10 years, Holman et al. [37] report risk reduction for myocardial infarction (MI) and all cause cardiovascular death after intensive glucose-lowering therapies although there was no data relating to heart failure.

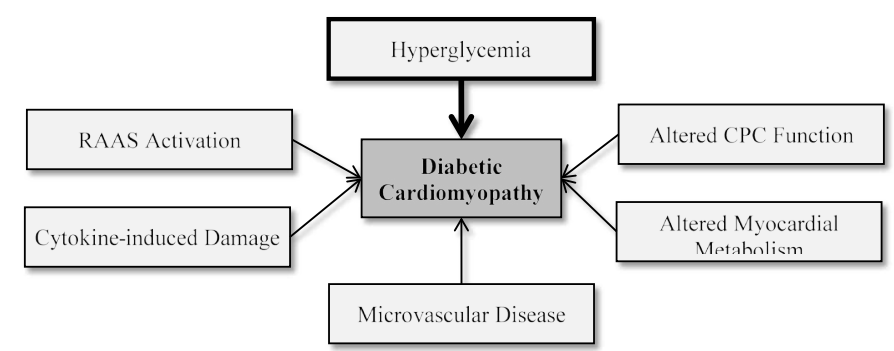

Figure 1. Pathophysiologic Mechanism of Diabetic Cardiomyopathy. (Adapted from Aneja et al., 2008, p. 750 [49]) 
Albakri A (2018) Diabetic cardiomyopathy: a review of literature on clinical status and meta-analysis of tissue doppler diagnostic method and the clinical value of intensive glycemic control

Hypertension: Hypertension (HT) has also been linked to increased risk of developing heart failure in patients with diabetic cardiomyopathy but its true effect in research findings remains conflicting. Hayat et al. [38] study on mechanism, diagnosis and treatment report independent association between HT and LV hypertrophy, diastolic dysfunction, HF and cardiovascular risk. The UKPDS trial [29] also associated acute reduction of blood pressure level obtained using pharmacotherapy (beta-blocker or Angiotensin Converting Enzyme [ACE] - Inhibitor) with reduced risk of $\mathrm{HF}$ than observed in control patients receiving less intensive blood pressure control. However, in long-term follow-up (10 years) the reduction of blood pressure did not yield risk reduction for MI or all-cause cardiovascular death.

Clinical presentation: Clinical presentation of diabetic cardiomyopathy has been classified into two distinct phases: (a) asymptomatic subclinical phase, and (b) clinically-evident (symptomatic) phase [39].

Asymptomatic Subclinical Phase: A unique feature of diabetic cardiomyopathy is a prolonged subclinical phase that is completely asymptomatic [10]. In most cases, this subclinical phase is detected with co-occurring CAD or HT. However, an important initial clinical sign is usually echocardiographically defined mild LV hypertrophy and diastolic dysfunction defined as the inability of the heart to relax appropriately between contractions. Diastolic dysfunction not accompanied by any other determinable clinical sign of cardiac disease such as depressed ventricular filling is often always the initial observable cardiac changes in DM patients [39]. Echocardiography characterizes diastolic dysfunction as increased time of isovolumic relaxation and Doppler flow changes in LV filling time. However, very subtle changes in diastolic function may require tissue Doppler echocardiography imaging and measurement of strain and strain rate [40,41]. Some DM patients in the subclinical phase may present with subtle signs of diabetic cardiomyopathy including decreased LV compliance or LV hypertrophy, or both. There may also be a prominent "a" wave and overactive or sustained cardiac apical pulse throughout systole [10].

Clinically Evident (Symptomatic) Phase: The second clinical phase is marked by clinically evident diabetic cardiomyopathy described by the presence of congestive heart failure (CHF) but in the absence of arteriosclerotic changes in coronary arteries and hypertension [39]. The development of systolic dysfunction, LV dilatation and symptomatic HF have also been reported. The jugular venous pressure may increase and apical pulse displaced downward to the left and some reported cases of systolic mitral murmurs [10]. These changes accompany a variety of electrocardiographic changes linked to diabetic cardiomyopathy in about two-thirds of patients without structural cardiac diseases. Prolonged QT may also suggest fibrosis. Frustaci et al. [42] suggest the deposition of collagen and its organization into irreversible fibrosis is the histological hallmark of diabetic cardiomyopathy. Since the definition of diabetic cardiomyopathy does not include co-occurring $\mathrm{CAD}$ or HT, there are no changes in perfusion or in the levels of atrial natriuretic peptide (ANP) until in the later stages of the disease when hypertrophy and fibrosis have become very noticeable [10].

Prognosis: A majority of studies on diabetic cardiomyopathy have focused on understanding pathogenesis or pathophysiology and clinical management strategies. However, some of these studies have mentioned, although in brief, prognostic predictors of patients with diabetic cardiomyopathy. Insulin resistance and metabolic syndrome have been reported to indicate poor prognosis since they are independent predictors of the development of HF in DM patients $[43,44]$. In support of these findings, Hassan et al. [45] examined a retrospective cohort of HF patients, and reported metabolic syndrome in $68 \%$. However, the study also reported lower mortality in patients with higher percentage of metabolic syndrome (44\%) compared to those with $58 \%$. Inflammatory markers such as C-reactive protein (CRP) and interleukin (IL)-6 and microalbuminuria, which are increased in DM patients with metabolic syndrome, suggest poor prognosis associated with the development of HF, and as such, cardiomyopathy may develop in pre-diabetic individuals [46]. Worsening glycemic control may also suggest unfavorable prognosis of diabetes and HF in patients with diabetic cardiomyopathy [9].

\section{Pathophysiology}

A precise understanding of the pathophysiologic mechanisms of diabetic cardiomyopathy remains tenuous. However, experimental animal models and human studies [11,16,47-54] have suggested several mechanisms underlying morphological and functional pathological changes in the development of diabetic cardiomyopathy. These mechanisms are highly interrelated despite different studies presenting them as separate topics. The goal of this review is to present a concise introductory description of the complex pathophysiologic mechanisms of diabetic cardiomyopathy as a road map for more detailed exploration. Whereas several mechanisms have been proposed, the common ones include hyperglycemia, renin-angiotensin-aldosterone system (RAAS) activation, cytokine-induced damage, microvascular disease, altered myocardial metabolism (increased free fatty acid [FFA]) utilization and altered cardiac progenitor cells (CPC) function (Figure 2).

Hyperglycemia has been repeatedly mentioned as an important factor in the pathophysiology of diabetic cardiomyopathy. It is implicated as a trigger for several adaptive and maladaptive changes observed in diabetic patients. Figure 2 illustrates the pathophysiologic processes triggered by hyperglycaemia.

Altered Myocardial Metabolism: In non-diabetic individuals, myocardial glucose and free fatty acids (FFA) oxidation provide about the same proportions of energy for cardiac contractility whereas in diabetic patients, the dominant source of energy is FFA. DM patients have a marked increase in the uptake and oxidation of FFAs in relation to myocardial glucose uptake, glycolysis and glucose oxidation [5557]. Reduced glucose metabolism is a consequence of depleted glucose transport proteins and FAA's inhibition of pyruvate dehydrogenase. The inhibition impairs the production of myocardial glucose and increases the levels of ceramide and toxic glycolytic intermediaries [58-60] resulting into increased myocyte apoptosis, impaired myocyte calcium

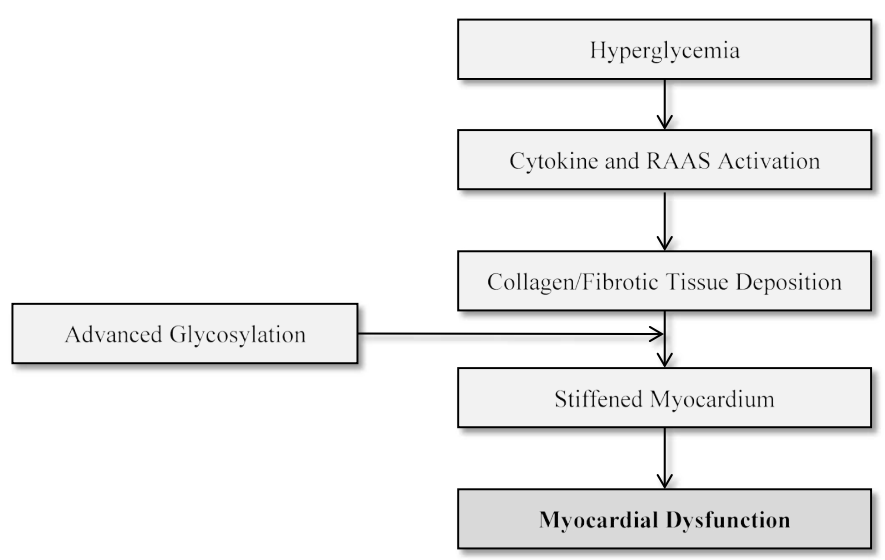

Figure 2. Effect of Glycaemia on Myocardial Dysfunction in Diabetic Cardiomyopathy Adapted from Aneja et al., 2008, p. 750 [49] 
Albakri A (2018) Diabetic cardiomyopathy: a review of literature on clinical status and meta-analysis of tissue doppler diagnostic method and the clinical value of intensive glycemic control

handling and impaired myocardial contractility [61-63]. Increased FFA oxidation is also associated with increased myocardial oxygen consumption, which is not accompanied by a relative increase in cardiac contractility suggesting reduced cardiac efficiency [56,64]. Figure 3 illustrates the role of increased FFA utilization in the development of diabetic cardiomyopathy.

Apoptotic and Necrotic Myocyte Death: Diabetic patients generally exhibit accelerated myocyte death through apoptosis and necrosis [49,50]. Many pathophysiologic mechanisms that increase fibrosis and myocardial inflammation could also accelerate proapoptotic signaling pathways or trigger necrosis signaling. Accelerated apoptotic and necrotic cell death has been observed in hearts of humans with T1DM and T2DM as well as in the hearts of diabetic rodent models [42,65-68]. Suggested mechanisms that may precede apoptotic effect of hyperglycemia include: (a) increased production of hyperglycemia-associated reactive oxygen species (ROS); (b) increased levels of circulating inflammatory cytokines and chemokines; (c) increased glycosylation and phosphorylation of p53; and (d) increased production of angiotensin II [67-69]. However, it is not clear whether accelerated myocyte apoptotic death is the cause or the effect of diabetic cardiomyopathy [49]. In addition to apoptosis, emerging evidence links other processes such as autophagy, mitophagy and PARP-dependent cell death pathways to myocyte necrosis during the clinical course of diabetic cardiomyopathy [70,71]. However, the role of autophagy and mitophagy in the pathophysiology of diabetic is an ongoing research area [50].

Myocardial Fibrosis and Necrosis: Myocardial fibrosis and collagen deposition are the primary histological changes observed in the diabetic myocardium [42,72]. Diabetes contributes to myocyte necrosis and fibrosis by triggering locally active RAAS and endothelium system $[73,74]$. Hyperglycemia-associated apoptotic myocyte death may cause interstitial collagen accumulation but not in significant amounts. On the other hand, myocyte necrotic death results into significant deposition of collagen in both diffuse and scattered patterns [65]. Collagen deposition in a diabetic myocardium may also result from impaired collagen degradation due to glycosylation of lysine residues on collagen [75]. The interaction of collagen interacts with

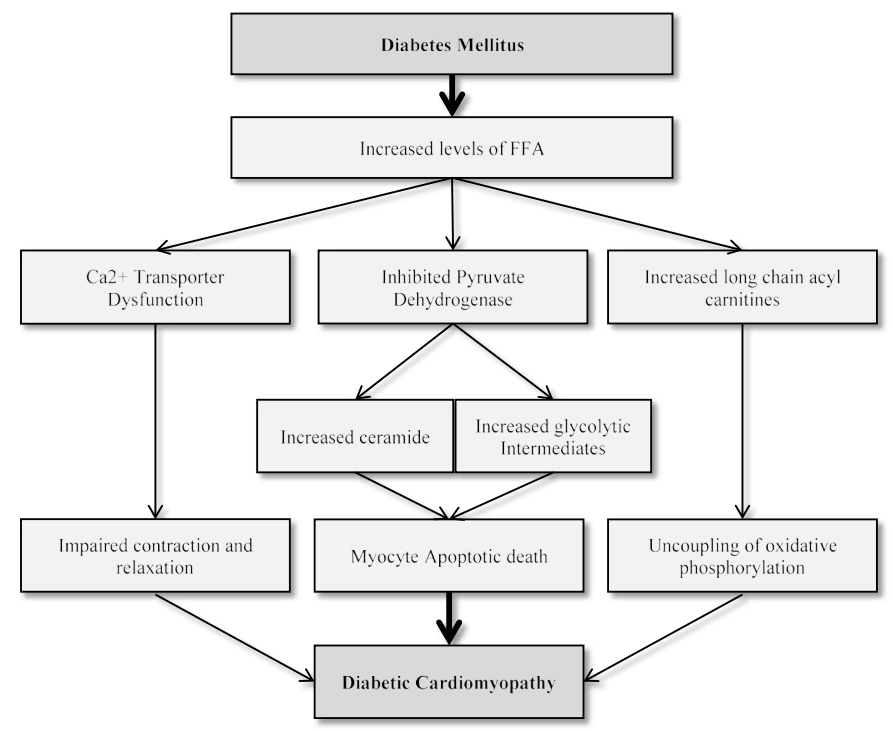

Figure 3. The Role of Increased FFA Utilization in Diabetic Cardiomyopathy (Adapted from Aneja et al., 2008, p. 750 [49]) glucose forms Schiff bases, which with time re-organize into glycated collagen (also known as Amadori products). Glycated collagen then undergoes further chemical modification to form advance glycation end-products (AGE), which contribute to myocardial stiffness and the development of atherosclerotic plaque $[73,75]$. Advance glycation endproducts may also contribute to myocyte damage through increasing intracellular oxidative stress [77]. These findings associate fibrosis and collagen deposition due to altered collagen structure and the formation of advance glycation end-products with altered myocardial reflectivity and LV dysfunction in diabetic cardiomyopathy.

Microvascular (Small Vessel) Disease: Structural and functional changes in microvascular architecture in diabetic patients have also been incriminated in the development of cardiomyopathy [48]. Biopsy studies report patients with diabetic cardiomyopathy have normal to mildly depressed LV systolic function, thickened capillary membrane, fibrosis surrounding arterioles, and smaller myocytes compared to nondiabetic subjects $[48,49]$. Diabetic patients with or without obstructive CAD and LV hypertrophy have reduced coronary blood flow reserve [49]. These findings suggest alterations in capillaries in diabetic patients may contribute to myocyte injury and interstitial fibrosis and eventually diabetic cardiomyopathy. Hamby et al. [78] conducted autopsy study on diabetic patients and observed an association between microvascular and myocardial diseases. Small coronary arteries of diabetic patients had endothelial and sub-endothelial fibrosis [78].

Despite these findings supporting association between microvascular changes and diabetic myocardium, they are insufficient to explain diffuse myocardial degeneration and interstitial fibrosis in patients with diabetic cardiomyopathy [48]. Further, there is no significant difference in the extent of microvascular disease or the density of micro-vessels between diabetic and non-diabetic patients [78]. Zhi et al. [79] also report no direct evidence of microvasculopathy as one of the etiologies of diabetic cardiomyopathy [79].

Altered Mitochondrial Function: Altered mitochondrial function has also been associated with the pathogenesis of diabetes and its complications in diabetes-affected tissues. In a recent review, Duncan [80] implicated altered mitochondrial metabolism as a pathogenic factor in the development of contractile dysfunction and ventricular failure in patients with diabetic cardiomyopathy [80]. However, it was Anderson and associates. [81,82], who provided the most compelling evidence of mitochondrial dysfunction in the myocardium of diabetic patients. They observed impaired mitochondrial respiratory capacity - increased mitochondrial oxidative stress and increased sensitivity to $\mathrm{Ca}^{2+}$ triggered opening of the mitochondrial permeability transitional pore in atrial tissues of T2DM patients.

Despite these recent studies, research evidence of mitochondrial dysfunction in rodent models had already existed for more than three decades [50]. In 1985, Kuo et al. [83] was the first to observe reduced state 3 mitochondrial respiration in hearts of obese and $\mathrm{T} 2 \mathrm{DM} \mathrm{db} / \mathrm{db}$ mice. In a review of literature on rodent models of diabetic cardiomyopathy, Bugger et al. [84] report impaired state 3 respiration, and abnormal mitochondrial oxidative stress and ultrastructure in experimental murine model with various degrees of obesity and types of diabetes: insulin-resistant, insulin-dependent and non-insulin dependent. The underlying pathological mechanism in altered mitochondrial function and morphology include oxidative damage, alterations in oxidative phosphorylation, impaired mitochondrial $\mathrm{Ca}^{2+}$ handling and alterations in cardiac insulin handling $[85,86]$. 
Albakri A (2018) Diabetic cardiomyopathy: a review of literature on clinical status and meta-analysis of tissue doppler diagnostic method and the clinical value of intensive glycemic control

\section{Diagnosis}

Diagnosis Status: Clinical diagnosis of diabetic cardiomyopathy has two important components: (a) the detection of myocardial abnormalities; and (b) the exclusion of other known causes of cardiomyopathies such as structural heart diseases and hypertension [49]. Still, diagnosis is problematic because of the lack of distinguishing pothognomic histologic changes or imaging characteristics specific to diabetic-associated alterations in cardiac functions. Although endomyocardial biopsy could differentiate diabetic cardiomyopathy from other cardiomyopathies or similar cardiac diseases such as hypertrophic cardiomyopathy or infiltrative cardiac diseases, it is rarely recommended because of its invasive nature [11]. However, myocardial fibrosis or collagen deposition remain an important histological feature of diabetic cardiomyopathy.

\section{Diagnostic Methods}

Non-Invasive Imaging: Non-invasive imaging is considered the mainstay of diagnosing diabetic cardiomyopathy because it is able to visualize and characterize myocardial dysfunction across the spectrum of its clinical manifestation. Echocardiography-defined cardiac dysfunction or morphological abnormalities in patients with overt HF often provide confirmatory diagnosis of diabetic cardiomyopathy. In the absence of symptoms in overt HF, the American College of Cardiology/American Heart Association (ACC/AHA) recommend imaging diagnosis [87]. Imaging diagnosis could be complemented by assessing circulating cardiac biomarkers produced by alterations in biochemical tissue composition [88].

Echocardiography: Echocardiography is an inexpensive noninvasive imaging tool for characterizing cardiac structure and function as well as enabling real time visualization of cardiac cycle [9]. It is the accepted imaging standard for the assessment of systolic and diastolic dysfunction in diabetic patients. Nevertheless, current clinical understanding of diabetic cardiomyopathy lack consensus on precise echocardiographic imaging criteria for the diagnosis of diabetic cardiomyopathy. However, the presence of hypertrophy (increase in LV mass) or diastolic dysfunction provides important supportive evidence for diagnosis. In particular, two recent reviews of echocardiographicdefined diagnosis of diabetic cardiomyopathy suggest imaging criteria should include one or both of the following features as listed in Table 3 [11,49]. Further, the criteria suggests conventional Doppler echocardiography may be sufficient but tissue Doppler imaging of mitral annular motion is of particular importance in confirming diagnosis.

Echocardiography images enable quantitative and qualitative assessment of cardiac function including LV geometry, regional wall motion, systolic/diastolic function and valvular anatomy and function. Two-dimensional (2D) echocardiography is the traditionally validated imaging method of choice for detecting and quantifying LV hypertrophy. However, because 2D echocardiography has important limitations is assessing DM patients exhibiting major distortion in LV geometry, a majority of existing studies have assessed LV hypertrophy and LV mass

Table 3. Suggested Imaging Criteria for Diagnosis of Diabetic Cardiomyopathy

\begin{tabular}{|l|l|l|}
\hline Criteria & Imaging Description & Imaging Technique \\
\hline & Evidence of cardiac hypertrophy & $\begin{array}{l}\text { Conventional echocardiography or } \\
\text { cardiac magnetic resonance imaging }\end{array}$ \\
\hline & $\begin{array}{l}\text { Evidence of LV diastolic } \\
\text { dysfunction with or without } \\
\text { systolic dysfunction; or Evidence } \\
\text { of left atrial enlargement }\end{array}$ & $\begin{array}{l}\text { Echocardiography: Transmitral Doppler } \\
\text { or Tissue Doppler, or provocative testing } \\
\text { (strain/strain rate imaging or stress } \\
\text { imaging). }\end{array}$ \\
\hline
\end{tabular}

using a combination of 2D and M-mode echocardiography. Although cardiac catheterization is considered the gold standard in assessing LV diastolic dysfunction, in diabetic patients, it is rarely considered [89]. Pulsed-wave Doppler echocardiography is thus the most clinically practical method for assessing diastolic dysfunction, which includes measurement of transmitral and pulmonary venous flow or velocities, and atrial volumes [90].

Cardiac Magnetic Resonance: Cardiac magnetic resonance imaging (CMRI) has been shown to have superior accuracy and reproducibility and thus has been considered the gold standard in assessing LV mass [91]. It is able to perform myocardial perfusion imaging (MPI) and assess myocardial flow reserve (MFR) [92]. Further, CMRI has been demonstrated as a useful imaging modality in accurately assessing diastolic function better than echocardiography [93]. However, the current use of CMRI is limited because of high cost, time constraints and the requirement for expertise assessment [9].

Cardiac Biomarkers: Assessment of circulating cardiac biomarkers produced by alterations in biochemical tissue composition may complement imaging findings in the diagnosis of diabetic cardiomyopathy [88]. The most recognized cardiac biomarker is brain natriuretic peptide (BNP) hormone produced in response to ventricular volume and pressure overload. A study examining BNP levels in relation to findings in echocardiography reports patients with diabetic cardiomyopathy have elevated levels of BNP accompanying LV dysfunction [94]. In a related study comparing BNP levels and echocardiographic findings, Epshteyn et al. [95] reported in patients with diabetic cardiomyopathy, BNP levels have a high positive predictive value for detecting LV dysfunction, (96\% with BNP levels > $90 \mathrm{pg} /$ $\mathrm{ml}$ ). However, although BNP levels may provide additional diagnostic information, it is a sensitive and specific marker for congestive heart failure but cannot reliably distinguish between systolic and diastolic heart failure, which limits its clinical use in the diagnosis of diabetic cardiomyopathy $[36,94]$.

Meta-Analysis of Diagnosis of Diabetic Cardiomyopathy: The search for studies on the diagnosis of diabetic cardiomyopathy was carried out on online databases: PubMed, EMBASE and Google Scholar. To find as many as possible published studies, a combination of wide search terms were used. This included diabetic cardiomyopathy, diabetes and heart failure, diabetes and depressed ventricular function, diabetes and LV hypertrophy. Studies were included if they: (a) used original data; (b) assessed LV function using echocardiography; and (c) included at least one of the following key diagnostic features represented in numerical or percentage form: diastolic dysfunction, impaired relaxation, pseudo-filling pattern, LV mass index, E/A or E/e' ratio, peak strain and strain rate. Titles and abstract of studies retrieved from online search as well as studies obtained from reviewing bibliographies suspected to meet the inclusion criteria were included for further review. Studies on experimental animal models and case series with less than ten patients (because of issues of statistical inadequacy) were excluded from this meta-analysis. Table 4 summarizes study features relevant to this meta-analysis.

Study Characteristics: A combined search on online databases, library and a review of bibliography returned more than 200 studies for further review. However, only fourteen (14) met the inclusion criteria and were included in this meta-analysis [25,96-108]. Table 4 provides a summary of the main diagnostic methods and features relevant the current analysis. The main echocardiographic methods used were conventional Doppler echocardiography to measure blood flow velocities in eight (57\%) of the studies [25,96-102] and tissue Doppler 
Albakri A (2018) Diabetic cardiomyopathy: a review of literature on clinical status and meta-analysis of tissue doppler diagnostic method and the clinical value of intensive glycemic control

Table 4. Summary of Key Diagnostic Features of Diabetic Cardiomyopathy

\begin{tabular}{|c|c|c|c|c|c|c|c|c|c|c|c|c|c|c|}
\hline $\begin{array}{l}\text { 1st Author [Ref. } \\
\#]\end{array}$ & $\begin{array}{c}\text { Year of } \\
\text { Publication }\end{array}$ & $\begin{array}{c}\text { No. of } \\
\text { Patients }\end{array}$ & $\begin{array}{l}\text { Men } \\
(\%)\end{array}$ & $\begin{array}{l}\text { Mean } \\
\text { Age }\end{array}$ & $\begin{array}{l}\text { Diagnostic } \\
\text { Method }\end{array}$ & $\begin{array}{c}\text { Diastolic } \\
\text { Dysfunction } \\
(\%)\end{array}$ & $\begin{array}{c}\text { Impaired } \\
\text { Relaxation } \\
(\%)\end{array}$ & $\begin{array}{c}\text { Pseudo- } \\
\text { filling } \\
\text { pattern } \\
(\%)\end{array}$ & $\begin{array}{c}\text { LV mass } \\
\text { index }(g / \\
\left.\mathbf{m}^{2}\right)\end{array}$ & $\begin{array}{l}\text { Mean } \\
\text { E/A } \\
\text { Ratio }\end{array}$ & $\begin{array}{l}\text { Mean } \\
\text { DT } \\
(\mathrm{ms})\end{array}$ & $\begin{array}{c}\text { E/e' } \\
\text { Ratio }\end{array}$ & $\begin{array}{c}\text { Peak } \\
\text { Strain } \\
(\%)\end{array}$ & $\begin{array}{c}\text { Strain } \\
\text { Rate } \\
\left(\mathbf{s}^{-1}\right)\end{array}$ \\
\hline $\begin{array}{l}\text { Devereux et al. } \\
{[25]}\end{array}$ & 2000 & 1810 & 32 & 60 & Doppler & NR & NR & NR & 85 & NR & NR & NR & NR & NR \\
\hline Khan et al. [96] & 2006 & 50 & 72 & 48 & Doppler & 60 & 36 & 24 & 83 & 0.76 & 260 & NR & NR & NR \\
\hline $\begin{array}{l}\text { Zabalgoitia et al. } \\
{[97]}\end{array}$ & 2001 & 86 & 52 & 46 & Doppler & 47 & 30 & 17 & 101 & NR & NR & NR & NR & NR \\
\hline From et al. [98] & 2010 & 1760 & 49 & 60 & Doppler & 23 & NR & NR & 97 & NR & 217 & 13.0 & NR & NR \\
\hline Poirier et al. [99] & 2001 & 46 & 100 & 52 & Doppler & 60 & 32 & 28 & 88 & 0.79 & 279 & NR & NR & NR \\
\hline Liu et al. [100] & 2001 & 616 & 25 & 58 & Doppler & NR & 50 & NR & 40 & 0.83 & 202 & NR & NR & NR \\
\hline Eren et al. [101] & 2004 & 24 & 37 & 51 & Doppler & 59 & 41 & 17 & 86 & 0.80 & 245 & NR & NR & NR \\
\hline Zarich et al. [102] & 1988 & 21 & 43 & 32 & Doppler & 29 & 20 & NR & NR & 1.24 & NR & NR & NR & NR \\
\hline Ha et al. [103] & 2007 & 53 & 51 & 53 & TDI & NR & NR & NR & & 1.10 & 193 & NR & NR & NR \\
\hline Fang et al. [104] & 2003 & 48 & 69 & 60 & TDI & NR & 53 & NR & 95 & 1.02 & NR & NR & 24.1 & 1.4 \\
\hline $\begin{array}{l}\text { Andersen et al. } \\
{[105]}\end{array}$ & 2003 & 32 & 56 & 53 & TDI & 43 & 28 & 15 & 99 & 1.10 & 198 & NR & NR & 1.2 \\
\hline Nakai et al. [106] & 2009 & 60 & 57 & 63 & TDI & NR & NR & NR & 115 & 0.80 & 243 & 12.9 & 23.1 & NR \\
\hline $\begin{array}{l}\text { Palmieri et al. } \\
{[107]}\end{array}$ & 2008 & 25 & 68 & 33 & TDI & 8 & 0 & 0 & NR & 1.50 & & 5.6 & 19.0 & 1.4 \\
\hline $\begin{array}{l}\text { Konduracka et al. } \\
{[108]}\end{array}$ & 2007 & 185 & 52 & 35 & TDI & 0 & 0 & 0 & NR & 1.30 & 191 & 7.5 & NR & NR \\
\hline Total/Average & & 4,816 & 55 & 50 & & 36.6 & 29.0 & 14.4 & 88.9 & 1.0 & 225.3 & 9.8 & 22.1 & 1.3 \\
\hline
\end{tabular}

(*E/e': Mitral early diastole velocity/ peak mitral annular velocity during early diastole: TDI: Tissue Doppler Imaging; E/A: Early ventricular filling wave (E)/Late ventricular filling wave (A); DT: E-wave Deceleration Time; STE: Speckle Tracking Echocardiography)

echocardiography to measure myocardial tissue velocities in six studies (43\%) of the studies [103-108]. Outcome analysis assessed LV diastolic dysfunction, impaired relaxation and pseudo-normal filling pattern using LV mass index $\left(\mathrm{g} / \mathrm{m}^{2}\right)$, E/A or E/e' ratio, deceleration time (DT), peak strain and strain rate.

Patient Characteristics: The combined patient population in the 14 studies was 4,816. Twelve (12) of the 14 studies included 4,606 asymptomatic, normotensive T2DM patients [25,96-106] while the remaining two included 201 uncomplicated or chronic (>10years) T1DM placed under intensive insulin therapy $[107,108]$. The mean age was 50 years, range 32 years [102] to 63 years [106] with an almost equal gender presentation ( $m e n=55 \%$, female $45 \%$ ). However, the duration of diabetes was not considered in this meta-analysis since several studies reported it did not influence the development or severity of ventricular systolic dysfunction $[96,97,99,102,106]$.

Echocardiographic Findings: The main diagnostic feature assessed in the 14 studies was LV diastolic dysfunction. Nine studies [96-99,101,102,105,107,108] reported 36.6\% (range 0\% [131] to $60 \%$ $[96,99])$ of the patients had LV diastolic dysfunction. In terms of patient characteristics, Konduracka et al. [131] studied patient sample of T1DM patients under intensive insulin therapy, while the rest of the studies investigated T2DM patients that were well associated with diabetic cardiomyopathy.

Based on the 14 studies, two key factors used to define ventricular diastolic dysfunction were impaired relaxation and pseudo-normal filling pattern accounting for $29.0 \%$ and $14.4 \%$ in ten $[96,97,99-102,104$, $105,107,108]$ and seven $[96,97,99,101,105,107,108]$ studies respectively. The ratio of ventricular filling (during rapid E-wave $[\mathrm{E}]$ and atrial contraction [A], E/A) and E-wave deceleration time [DT]) were also used to determine diastolic dysfunction, defined as normal diastolic (E/A >1, DT $160 \sim 240 \mathrm{~ms}$ ); impaired relaxation (E/A $<1$. DT $>240 \mathrm{~ms}$ ), and pseudo-normal filling (E/A $>1$, Decreased ratio by $>25 \%$ during Valsalva maneuver relative to baseline) [119]. The mean E/A ratio in 11 studies [96,99-108] was one (1.0) and DT in nine of the studies [96,98$101,103,105,106,108]$ was $225.3 \mathrm{~ms}$.
In addition to $\mathrm{E} / \mathrm{A}$ ratio, tissue Doppler imaging assessed $\mathrm{E} / \mathrm{e}$ ' ratio (mitral early diastole velocity/ peak mitral annular velocity during early diastole). E/e' ratio $>15$ suggests diastolic dysfunction [98]. Finally, using tissue Doppler imaging, peak strain and strain rate was assessed to detect diastolic dysfunction $[104,105,107]$. The mean peak strain in three studies using tissue Doppler imaging [104,106,107] was $22.1 \%$ and strain rate was 1.3/s. On the other hand, LV mass index was used to assess LV hypertrophy, a key feature of diabetic cardiomyopathy, defined as LV mass $>131 \mathrm{~g} / \mathrm{m}^{2}$ in men and $>100 \mathrm{gm}^{2}$ in women [104].

\section{Discussion}

A range of diagnostic methods may help to characterize and detect morphological and functional alterations in the normal cardiac function in many cardiomyopathy phenotypes as well as in other cardiac conditions. However, none of these alterations is specific to diabetic cardiomyopathy [49]. The assessment of LVEF is a wellestablished standard in characterizing LV dysfunction in many cardiac conditions but it has a limited use in diabetic cardiomyopathy because alterations in pre- and afterload reduce its sensitivity more so in HF patients with preserved ejection fraction [9]. Further, leading national heart associations and cardiologists societies such as AHA and ESC have not provided specific guidelines for diagnosis of diabetic cardiomyopathy. This meta-analysis aimed to determine specific alterations in the LV myocardium that could improve the diagnosis of diabetic cardiomyopathy.

In the present findings, a combination of LV functional and morphological indices could support the diagnosis of diabetic cardiomyopathy. Impaired relaxation and pseudo-filling LV diastolic pattern are described as key functional diagnostic characteristics. The key variables to demonstrate impaired relaxation and pseudo-filling LV diastolic pattern are: (a) early ventricular filling wave (E-wave) and the late ventricular filling wave (A-wave) reported as E/A ratio; (b) E-wave peak velocity (E)' and (c) E-wave deceleration time (DT) as measured by Tissue Doppler Imaging (TDI). These variables categorize diastolic 
Albakri A (2018) Diabetic cardiomyopathy: a review of literature on clinical status and meta-analysis of tissue doppler diagnostic method and the clinical value of intensive glycemic control

function into four patterns; (a) normal; (b) impaired relaxation; (c) pseudo-normal, or (d) restrictive pattern. An important limitation of impaired relaxation defined as E/A $<1$, DT $>240 \mathrm{~ms}$ is that it is nonspecific to diabetic cardiomyopathy because it is a characteristic shared by several other cardiac conditions such as microvascular myocardial ischemia, hypertension or LV overload $[88,96]$. However, the use of Valsalva maneuver improves the diagnostic value of pseudo-filling pattern, which is defined as (E/A $>1$, decreased ratio by $>25 \%$ during Valsalva maneuver relative to baseline.

In the present findings, diastolic dysfunction is also detectable by TDI assessed as a ratio of mitral early diastole velocity/ peak mitral annular velocity during early diastole $\left(\mathrm{E} / \mathrm{e}^{\prime}\right)>15$. Further, the findings suggest TDI-based strain and strain rate analysis is a promising diagnostic method for diabetic cardiomyopathy but its use is limited since it is an emerging research area. In addition to functional LV parameters, LV hypertrophy (LV mass index $>131 \mathrm{~g} / \mathrm{m}^{2}$ for men and $100 \mathrm{~g} / \mathrm{m}^{2}$ for women) and atrial enlargement are key morphological features supporting the diagnosis of diabetic cardiomyopathy. However, a paucity of studies specifically investigating atrial enlargement means insufficient evidence of its true value in the diagnosis of diabetic cardiomyopathy [49].

The present findings are consist with proposals by Aneja et al. [49] and Mehta et al. [11] that the presence of LV hypertrophy and/ or LV diastolic dysfunction are the key characteristic features of diabetic cardiomyopathy. However, whereas the present findings suggest functional alterations could support the diagnosis of diabetic cardiomyopathy, Aneja et al. [49] proposed that they are non-specific. Instead, the two proposals [11,49] suggest morphological characteristics, particularly LV hypertrophy, is the hallmark of diagnostic characteristic in patients with diabetic cardiomyopathy. In addition, LV hypertrophy had also been shown to be readily demonstrated by conventional echocardiography or CMR imaging modalities. In support, several large-scale population-based heart studies - Framingham Heart Study (FHS) [90], Cardiovascular Health Study (CHS) [22] and Strong Heart Study (SHS) [25] - have reported significant increases in LV mass in diabetic patients. According to Aneja et al. [49] proposed diagnostic guidelines, increases in LV mass (indicating LV hypertrophy) supports the diagnosis of diabetic cardiomyopathy.

Whereas echocardiographic-defined LV ejection fraction (LVEF) is the well-established standard for assessing LV dysfunction, alterations in pre-load and after-load can influence LVEF reducing its sensitivity for assessing contractile function in HF patients with normal ejection fraction [9]. The present findigns reveal that tissue Doppler Imaging (TDI) echocardiography enables better detection of subtle regional abnormalities in LV function. TDI has also been demonstrated to assess myocardial strain and identify global and regional abnormalities in the diabetic myocardium [9]. TDI is different from conventional Doppler because it uses a filter to eliminate high velocity and low amplitude signals reflection from blood cells and thus enables visualization and analysis of low velocity and high amplitude tissue signals. Further, TDI enables assessment of strain and strain rate as dimensionless indices of changes in length signifying tissue deformation [90].

\section{Summary of Findings}

Diagnosis of diabetic cardiomyopathy is challenging because of the lack of pothognomic imaging characteristics. However, growing evidence suggests alterations in LV functions such as impaired diastolic function and pseudo-filling patterns as well as LV hypertrophy could support diagnosis. Conventional echocardiography is useful for assessing changes in LV mass (hypertrophy), tissue Doppler imaging is a better echocardiography imaging modality for assessing LV dysfunction in diabetic cardiomyopathy since it measures hemodynamic alterations: blood flow and myocardial tissue velocities, or myocardial strain and strain rate. While atrial enlargement could also support diagnosis of diabetic cardiomyopathy, its use is undermined by insufficient evidence.

Clinical management: Unlike other primary forms of cardiomyopathy such as dilated cardiomyopathy, hypertrophic cardiomyopathy and restrictive cardiomyopathy, diabetic cardiomyopathy lacks specific clinical guidelines. However, the American College of Cardiology/American Heart Association Task Force (ACC/AHA) recommends treatment of diabetes should include management diabetes and associated risk factors, and the treatment of heart failure.

Therapeutic targets: The ACC/AHA recommend the main therapeutic targets in the clinical management of diabetic cardiomyopathy are (a) lifestyle modification; (b) regulation of blood glucose (glycaemia); (c) modification of risk factors for the development of cardiovascular diseases; and (d) the treatment of heart failure [47].

\section{Management Methods}

Lifestyle Modification: Lifestyle has been associated with the development or the exacerbation of diabetes, and this association has isolated lifestyle modification as an important prophylactic management strategy for diabetic cardiomyopathy [47]. The recommended lifestyle modifications mostly include smoking cessation, healthy eating habits, and aerobic exercise (intendent to reduce body weight). Several animal models and human studies [95,109-112] have demonstrated that reduction in body weight and increased aerobic exercise significantly reduce the incidence of T2DM and diabetic cardiomyopathy.

Glycemic Control: The control of hyperglycemia has important clinical benefits in the management of diabetic cardiomyopathy. Several studies have suggested hyperglycemia induces increased production and oxidation of FFA and growth factors leading to abnormalities in substrate supplies and utilization. Thus, glycemic control is the most basic and important strategy to prevent the development of diabetic cardiomyopathy [48] since it reduces the risk of cardiovascular events including myocardial infarction, stroke and the likelihood of developing diabetic cardiomyopathy. Although current management of diabetes uses a range of medication - metformin, sulfonylureas, insulin and other modern drugs including GLP1 agonists and antagonists of DPP4, these medications have important limitations for diabetic patients with co-occurring heart failure. Metformin for instance introduces the risk of lactic acidosis in HF patients but the risk is not significant [113]. However, Metformin has been demonstrated to have a preventive effect against the development of diabetic cardiomyopathy in murine models [101] as well as reduces mortality and hospital admission [115,116]. Insulin treatment has also been used as a glycemic control strategy in high-risk diabetic patients [47].

Heart Failure Treatment: For DM patients with depressed LV function and symptomatic heart failure, the 2013 European Society of Cardiologists (ESC) on diabetes, pre-diabetes and cardiovascular diseases recommend three neuro-hormonal antagonists: ACEinhibitor, angiotensin receptor blocker (ARB) and mineralocorticoid receptor antagonist (MRA). These are the leading pharmacologic agents in the treatment of HF and depressed LVEF, which includes diabetic patients. These medications should be used concomitantly with diuretic to relieve congestion [47]. 
Albakri A (2018) Diabetic cardiomyopathy: a review of literature on clinical status and meta-analysis of tissue doppler diagnostic method and the clinical value of intensive glycemic control

Angiotensin-Converting Enzyme Inhibitors/Angiotensin Receptor Blockers: Angiotensin-converting enzyme (ACE) - inhibitors are usually indicated in patients with T2DM and HF to improve symptoms and survival [117-120]. Angiotensin receptor blockers (ARB) provide equivalent beneficial effect to that provided by ACEinhibitors and thus ARB could be used as an alternative for patients intolerant to ACE-inhibitors [121]. However, the use of ACE-inhibitors and $\mathrm{ARBs}$ in $\mathrm{DM}$ patients requiring monitoring of kidney function and potassium because of the frequent occurrence of nephropathy [47].

Beta-Blockers: Beta-blockers are the accepted standard pharmacologic care for patients with systolic heart failure $[109,110]$. In a sub-group analysis of the 1999 Metoprolol CR/XL Randomized Intervention Trial in Congestive Heart Failure (MERIT-HF), Deedwania et al. [124] observes beta-blockers had a protective effect on mortality and hospital admissions, and improve survival rate without significant differences between T2DM patients and nondiabetic patients. The main recommended beta-blocker medications in HF and T2DM patients include metoprolol [124], Bisoprolol in the Cardiac Insufficiency Bisoprolol Study (CIBIS II) [125], Carvedilol in the Carvedilol Prospective Randomized Cumulative Survival (COPERNICUS) [126], and Carvedilol or Metoprolol European Trial (COMET) [127]. Administration of beta-blockers in T2DM patients should be monitored because of the risk of adverse effects including negative metabolic effect such as hypoglycemia, dyslipidemia and reduced insulin sensitivity $[128,129]$.

Mineralocorticoid Receptor Antagonists: Mineralocorticoid receptor antagonists (MRA) is recommended for all symptomatic patients with depressed LV function with or without ACE-Inhibitor/ ARB treatment to reduce the risk of HF hospitalization and premature death [16]. The effect of MRA does not differ between patients with or without T2DM and HF [130]. However, monitoring of kidney function and potassium is mandatory due to increased risk of nephropathy in DM patients [47].

Diuretics: Diuretics are usually recommended for relief of dyspnea and edema in HF patients with fluid retention with or without depressed ejection fraction. However, there is no demonstrated protective effect against mortality or morbidity. In diabetic patients, loop diuretics are preferred over ACE-inhibitors/ARB due to their superior effect on glycemic profile [47].
Calcium Channel Blockers: Intracellular calcium retention depletes high-energy phosphate stores and impairs cardiac function. Calcium channel blockers are indicated to reverse intracellular calcium deficits and prevent against diabetic-induced myocardial changes [47]. Verapamil has been reported to improve contraction and rate of relaxation as well as improve altered myofibrillar. ATPase activities and reticular calcium pump activities [131].

Meta-analysis of Clinical Management: Hyperglycemia has been recognized as an important pathogenic factor contributing to altered myocardial metabolism, which precedes the development of diabetic cardiomyopathy. However, the efficacy of glycemic control in the management of diabetic cardiomyopathy has only been demonstrated in murine models but remains inconclusive in human studies. This meta-analysis reviews five large randomized control trials: ACCORD [132], UKPDS [133], ADVANCE [134], PROactive [135], and VADT [136] that compared standard and intensive glycemic control in the management of cardiovascular events in diabetic patients. The aim is to determine whether intensive glycemic control compared to standard therapy conveys a protective effect against cardiovascular events and cardiovascular deaths. Table 5 provides a summary of the main features of the five RCTs in relation to glycemic control in diabetic patients.

Study Characteristics: Five large randomized controlled trials [132-136] with long follow-up periods were included in this metaanalysis. The combined total patient population was 33,040 (mean age $=60.7$ years; female $31.8 \%$ ). The median follow-up was 5.3 years, range 10.1 [133] to 2.1 years [135]. The mean duration of diabetes was 8.6 years in four RCTS [132,134-136]. The UKPDS trial [133] recruited patients with newly diagnosed diabetes. Standard therapy used standard medication based on local guidelines [132,134,135], conventional diet therapy [133] or half the dose of intensive glycemic control [136]. Intensive glycemic control on the other hand used a combination of medication including insulin, metformin, sulfonylurea, thiazolidinedione, glinide, rosiglitazone or glimepiride.

\section{Clinical Outcomes}

The main clinical endpoints of interest were the reduction in the concentration of glycated hemoglobin between baseline and followup, cardiovascular deaths, and all-cause mortality. Intensive glycemic control reduced the concentration of glycated hemoglobin $\left(\mathrm{HbA}_{1 \mathrm{c}}\right)$

Table 5. Comparing Standard and Intensive Glycemic Control in Diabetic Patients

\begin{tabular}{|c|c|c|c|c|c|c|c|}
\hline \multicolumn{2}{|l|}{ Trial [Ref \#] } & ACCORD [132] & UKPDS [133] & ADVANCE [134] & PROactive [135] & VADT [136] & Sum/Average \\
\hline \multicolumn{2}{|l|}{ Study Period } & $2001-2005$ & $1977-1997$ & $2002-2007$ & 2001-2002 & $2000-2007$ & -- \\
\hline \multicolumn{2}{|l|}{ No. of Patients } & 10,251 & 4,620 & 11,140 & 5,238 & 1,791 & 33,040 \\
\hline \multicolumn{2}{|l|}{ Mean Age (years) } & 62.2 & 53.0 & 66.0 & 61.8 & 60.4 & 60.7 \\
\hline \multicolumn{2}{|l|}{ Female (\%) } & 38 & 41 & 43 & 34 & 3 & 31.8 \\
\hline \multicolumn{2}{|l|}{ Median Follow up (yrs.) } & 3.5 & 10.1 & 4.3 & 2.9 & 5.6 & 5.3 \\
\hline \multicolumn{2}{|l|}{ Mean Duration of Diabetes (yrs.) } & 10 & ND & 5.0 & 8.0 & 11.5 & 8.6 \\
\hline \multirow[b]{2}{*}{ Glycemic Control (Strategies) } & Standard & Standard medication & $\begin{array}{c}\text { Conventional diet } \\
\text { therapy }\end{array}$ & Standard medication & Standard medication & $\begin{array}{c}\text { Half-dose } \\
\text { intensive therapy }\end{array}$ & - \\
\hline & Intensive & $\begin{array}{l}\text { Metformin, insulin, } \\
\text { sulfonylurea, } \\
\text { thiazolidinedione }\end{array}$ & $\begin{array}{l}\text { Metformin, insulin } \\
\text { or sulfonylurea }\end{array}$ & $\begin{array}{c}\text { Glicazide, metformin, } \\
\text { glinide, thiazolidinedione } \\
\text { or insulin }\end{array}$ & $\begin{array}{c}\text { Metformin, } \\
\text { sulfonylurea, insulin } \\
\text { or a combination }\end{array}$ & $\begin{array}{l}\text { Metformin, } \\
\text { rosiglitazone, or } \\
\text { glimepiride }\end{array}$ & - \\
\hline \multicolumn{2}{|l|}{$\mathrm{HbA}_{1 \mathrm{c}}(\%)$ (Baseline) } & 8.3 & 7.1 & 7.5 & 7.8 & 9.4 & 8.0 \\
\hline \multirow{2}{*}{$\mathrm{HbA}_{1 \mathrm{c}}(\%)$ (Follow-up) } & Standard & 7.5 & 7.9 & 7.3 & 7.6 & 8.4 & 7.7 \\
\hline & Intensive & 6.4 & 7.0 & 6.8 & 7.0 & 6.9 & 6.8 \\
\hline \multirow[t]{2}{*}{ Cardiovascular Mortality } & Standard & 1.8 & -- & 4.6 & -- & 3.7 & 3.4 \\
\hline & Intensive & 2.6 & -- & 3.8 & -- & 4.5 & 3.6 \\
\hline \multirow{2}{*}{ All-cause Mortality } & Standard & 4.0 & 16.2 & 8.5 & 24.6 & 10.6 & 12.8 \\
\hline & Intensive & 5.0 & 19.5 & 7.3 & 23.6 & 11.4 & 13.4 \\
\hline
\end{tabular}

( $\boldsymbol{H b}_{1}$ : Glycated Hemoglobin; $\boldsymbol{N D}$ : Newly Diagnosed). 
Albakri A (2018) Diabetic cardiomyopathy: a review of literature on clinical status and meta-analysis of tissue doppler diagnostic method and the clinical value of intensive glycemic control

from baseline (8.0\%) to follow-up (6.8\%) compared to standard therapy (7.7\%). However, intensive glycemic control had comparable rates of cardiovascular and all-cause mortality to standard therapy $(3.6 \%$ vs. $3.4 \%)$ and $(13.4 \%$ vs. $12.8 \%)$ respectively. Intensive glycemic control significantly reduced the concentration of glycated hemoglobin with no significant increase in the risk of death. However, the five RCTs did not directly compare glycemic control in patients with diabetic cardiomyopathy nor assessed the effect on ventricular systolic dysfunction and heart failure.

\section{Discussion}

Glycemic control has been the mainstay of treatment in patients with diabetes mellitus. Several trials have demonstrated that glycemic control also inhibits the progression of microvascular diseases $[29,137]$ but its effect on macrovascular diseases remains unclear. However, epidemiological studies $[29,138,139]$ and small short-term trials $[140,141]$ have had inconsistent findings on the association between glycemic control and cardiovascular diseases some indicating beneficial effects while others indicating adverse effects. Since CVD is a serious complication and a leading cause of death in diabetic patients, its effective management is important to improve survival. In relation, the aim of the present meta-analysis was to determine the effect of intensive glycemic control compared to standard control on patients with diabetic cardiomyopathy.

The present analysis finds intensive glycemic control compared to standard glycemic control has beneficial protective effect against the progressive development of diabetic cardiomyopathy. According to Mishra et al. [48], intensive glycemic control reduces hyperglycemia, which is a key pathologic factor in diabetic cardiomyopathy because it contributes to apoptosis of cardiac myocytes and increases the production and oxidation of FFA and growth factors, which contribute to the pathophysiology of diabetic cardiomyopathy by interfering with substrate supply and utilization [36]. In support, Holman et al. [37] associate the reduction of Hba levels with improvement in both systolic function and systolic strain as measured by TDI. The study concludes that intensive glycemic control significantly reduces the concentration of glycated hemoglobin with no significant increase in the risk of death. Although glycemic control is an important prophylactic therapy, for DM patients with significantly depressed LV function and symptomatic heart failure, traditional HF treatment using pharmacotherapy (ACEinhibitors/ARB, beta-blockers, diuretics and calcium channel blockers), device therapy (ICD) or heart transplantation is recommended $[47,121,109,124]$.

All the five RCTs strongly demonstrate the therapeutic value of intensive glycemic control in reducing the concentration of glycated hemoglobin (a key etiologic feature in the development of diabetic cardiomyopathy). However, the five RCTs only assessed intensive glycemic control versus standard glycemic control in diabetic patients. The RCTs did not specifically identify or assess the effect of intensive glycemic control on diabetic patients with cardiomyopathy nor assessed the effect on ventricular systolic dysfunction. Furthermore, most current studies on glycemic control in patients with diabetic cardiomyopathy rely on experimental murine models and the application of the findings to human studies are still ongoing. Thus, there is a need for additional studies to determine the therapeutic value of intensive glycemic control in the management of patients with diabetic cardiomyopathy.

\section{Summary of Findings}

Hyperglycemia is one of the pathophysiologic mechanisms of diabetic cardiomyopathy. It results in apoptotic and necrotic myocyte death causing increased production and oxidation of FFA and growth factors, which contribute to ventricular dysfunction. Hyperglycemic control inhibits this pathophysiologic mechanism to convey a protective effect against the development of diabetic cardiomyopathy. However, although intensive glycemic control has superior effect in the reduction in the concentration of glycated hemoglobin compared to standard glycemic control, its clinical efficacy in preventing systolic dysfunction in diabetic patients has inconsistent results.

\section{Conclusion}

Diabetic cardiomyopathy is a heart muscle disease characterized by structural and functional abnormalities in the myocardium occurring in the absence of hypertension and/or structural heart disease in patients with diabetes. These abnormalities lead to systolic heart failure, which is one of the leading causes of diabetic-related morbidity and mortality. The prevalence of diabetic cardiomyopathy ranges between $1 \%$ and $4 \%$ in the general population and increases to $12 \%$ in diabetic patients. Diabetic patients with hyperglycemia and hypertension are at a higher risk of developing myocardial dysfunction. Clinically, diabetic cardiomyopathy has a long asymptomatic subclinical phase usually detected with concomitant coronary artery disease or hypertension, and a clinically distinct symptomatic phase, described as congestive heart failure in the absence of hypertension and coronary atherosclerosis. The main predictors of poor prognosis are worsening glycemic control (or insulin resistance), high concentration of inflammatory markers (C-reactive protein, interleukin (IL)-6 and microalbuminuria), the presence of metabolic syndrome and the development of heart failure. Key pathophysiologic mechanisms include altered myocardial metabolism, mitochondrial dysfunction, myocardial fibrosis, microvascular disease, and myocyte apoptosis or necrosis. Preceding these mechanisms is a series of maladaptive stimuli triggered by hyperglycemia. Diagnosis requires the evidence of echocardiographic-defined LV hypertrophy (increase in LV mass) and/or diastolic dysfunction (impaired relaxation and pseudofilling pattern) or atrial enlargement. Finally, treatment depends on management of diabetes mainly through lifestyle modification and intensive glycemic control. The other treatment option is management of heart failure using conventional heart failure pharmacotherapy.

\section{References}

1. Boudina S, Abel ED (2007) Diabetic cardiomyopathy revisited. Circulation 115: 3213 3223 .

2. Lorenzo-Almoros A (2017) Diagnostic approaches for diabetic cardiomyopathy Cardiovascular Diabetology 16: 28.

3. Leyden E (1881) Asthma and diabetes mellitus. Zeutschr. Klin. Med 3: 358-364.

4. Mayer J (1888) Ueber den zusammenhang des diabetes mellitus miterkrankungen des herzens. Zeutschr. Klin. Med 14: 212-239.

5. Lundbaek K (1954) Diabetic angiopathy: A specific vascular disease. The Lancet 263 377-379.

6. Lundbaek K (1969) Is there a diabetic cardiopathy? In: Schettler G. (ed.), "Pathogenetische faktoren des myokardinfarkts". Schattauer, Stuttgart: 63-71

7. Rubler S, Dlugash J, Yuceoglu YZ, Kumral T (1972) New type of cardiomyopathy associated with diabetic glomerulosclerosis. American Journal of Cardiology 30: 595602 .

8. Richardson P, McKenna W, Bristow M (1996) Report of the 1995 World Health Organization/International Society and Federation of Cardiology Task Force on the definition and classification of cardiomyopathies. Circulation 93: 841-842.

9. Asghar O, Al-Sunni A, Khavandi K, Khavandi A (2009) Diabetic cardiomyopathy Clinical Science 116: 741-760.

10. Mardikar HM, Admane P (2010) Diabetic Cardiomyopathy: A non-coronary complication of Diabetes Mellitus. Medicine Update 20: 358-360. 
Albakri A (2018) Diabetic cardiomyopathy: a review of literature on clinical status and meta-analysis of tissue doppler diagnostic method and the clinical value of intensive glycemic control

11. Mehta CN (2012) Diabetic Cardiomyopathy: Insights into Pathogenesis, Diagnostic Challenges, and Therapeutic Options. International Journal of Pharmaceutical Sciences and Research 3: 3565.

12. Westermeier F, Riquelme JA, Pavez M (2016) New molecular insights of insulin in diabetic cardiomyopathy. Frontiers in Physiology 7

13. Bell DS (2003) Heart failure: the frequent, forgotten, and often fatal complication of diabetes. Diabetic Care 26: 2433-2441.

14. Fein FS, Sonnenblick EH (1994) Diabetic cardiomyopathy, Cardiovascular Drugs Therapy, 8, 65-73

15. Litwin, S. E. (2013). Diabetes and the heart: Is there objective evidence of a human diabetic cardiomyopathy? Diabetes 62: 3329-3330.

16. Miki T, Yuda S, Kouzu H, Miura T (2013) Diabetic cardiomyopathy: Pathophysiology and clinical features. Heart Failure Reviews 18: 149-166.

17. Thrainsdottir IS, Aspelund T, Thorgeirsson G, Gudnason V (2005) The association between glucose abnormalities and heart failure in the population-based Reykjavik study. Diabetes Care 28: 612-616.

18. Bertoni AG, Hundley WG, Massing MW (2004) Heart failure prevalence, incidence, and mortality in the elderly with diabetes. Diabetes Care 27: 699-703

19. Reis SE, Holubkov R, Edmundowicz D (1997) Treatment of patients admitted to the hospital with congestive heart failure: specialty-related disparities in practice patterns and outcomes. Journal of American College of Cardiology 30: 733-738.

20. Cohen-Solal A, Beauvais F, Logeart D (2008) Heart failure and diabetes mellitus: epidemiology and management of an alarming association. Journal of Cardiac Failure 14: 615-625.

21. Amato L, Paolisso G, Cacciatore F (1997) Congestive heart failure predicts the development of non-insulin-dependent diabetes mellitus in the elderly. The Osservatorio Geriatrico Regione Campania Group. Diabetes Metabolism 23: 213-218.

22. Kannel WB, Hjortland M, Castelli WP (1974) Role of diabetes in congestive heart failure: The Framingham study. American Journal of Cardiology 34: 29-34

23. Bertoni AG, Tsai A, Kasper EK (2003) Diabetes and idiopathic cardiomyopathy: A nationwide case-control study. Diabetes Care 26: 2791-2795.

24. Gottdiener JS, Arnold AM, Aurigemma GP (2000) Predictors of congestive heart failure in the elderly: The Cardiovascular Health Study. Journal of American College of Cardiology 35: 1628-1637.

25. Devereux RB, Roman (2000) Impact of diabetes on cardiac structure and function: The strong heart study. Circulation 101: 2271-2276.

26. Bertoni AG, Goff DC, D’Agostino RB (2006) Diabetic cardiomyopathy and subclinica cardiovascular disease: The Multi-Ethnic Study of Atherosclerosis (MESA). Diabetes Care 29: 588-594.

27. Nichols GA (2001) Congestive heart failure in Type 2 Diabetes: Prevalence, incidence, and risk factors. Diabetes Care 24: 1614-1619.

28. Aronow WS, Ahn C (1999) Incidence of heart failure in 2737 older persons with and without diabetes mellitus. Chest 115: 867-868.

29. Stratton IM (2000) Association of glycaemia with macrovascular and microvascular complications of type 2 diabetes (UKPDS 35): Prospective observational study. British Medical Journal 321: 405-412.

30. Cheung N, Wang JJ (2008) Diabetic retinopathy and risk of heart failure. Journal of American College of Cardiology 51: 1573-1578.

31. Cheung N (2007) Retinal arteriolar narrowing and left ventricular remodeling: The multi-ethnic study of atherosclerosis. Journal of American College of Cardiology 50: $48-55$.

32. The SOLVD Investigators (1991) Effect of enalapril on survival in patients with reduced left ventricular ejection fractions and congestive heart failure. New England Journal of Medicine 325: 293-302.

33. Komajda M, Wimart (1994) The ATLAS study (Assessment of Treatment with Lisinopril and Survival); Justification and objectives. Arch. Mal. Coeur Vaiss 87: 45-50.

34. McKelvie RS (1999) Comparison of candesartan, enalapril, and their combination in congestive heart failure: randomized evaluation of strategies for left ventricular dysfunction (RESOLVD) pilot study. The RESOLVD Pilot Study Investigators. Circulation 100:1056-1064.

35. Andersen NH (2006) Effects of blood pressure lowering and metabolic control on systolic left ventricular function in Type II diabetes mellitus. Clinical Science 111: 5359.
36. Fang ZY (2005) Determinants of subclinical diabetic heart disease. Diabetologia 48: 394-402.

37. Holman RR (2008) Long-term follow-up after tight control of blood pressure in type 2 diabetes. New England Journal of Medicine 359: 1565-1576.

38. Hayat SA, Patel B, Khattar (2004) Diabetic cardiomyopathy: Mechanisms, diagnosis and treatment. Clinical Science 107: 539-557.

39. Karnafel W (2000) Diabetic cardiomyopathy. Pathophysiology and clinical implications. Przeglad Lekarski 57: 9-11.

40. Fang ZY, Prins JB (2004) Diabetic cardiomyopathy: Evidence, mechanisms, and therapeutic implications. Endocrine Reviews 25: 543-567.

41. Carugo S (2001) Progression of functional and structural cardiac alterations in young normotensive uncomplicated patients with type 1 diabetes mellitus. Journal of Hypertension 19: 1675-1680.

42. Frustaci A (2000) Myocardial cell death in human diabetes. Circulation Research 87 $1123-1132$

43. Ingelsson E (2006) Metabolic syndrome and risk for heart failure in middle-aged men Heart 92: 1409-1413.

44. Ingelsson E (2005) Insulin resistance and risk of congestive heart failure. Jama 294 334-341.

45. Hassan SA (2008) The metabolic syndrome and mortality in an ethnically diverse heart failure population. Journal of Cardiac Failure 14: 590-595.

46. Bahrami H (2008) Novel metabolic risk factors for incident heart failure and thei relationship with obesity: the MESA (Multi-Ethnic Study of Atherosclerosis) study. Journal of the American College of Cardiology 51: 1775-1783.

47. Trachanas K (2014) Diabetic cardiomyopathy: from pathophysiology to treatment Hellenic Journal of Cardiology 55: 411-421.

48. Mishra TK (2005) Diabetic cardiomyopathy: Evidences, pathophysiology, and therapeutic considerations. Journal Indian Academy of Clinical Medicine 6: 313.

49. Aneja A (2008) Diabetic cardiomyopathy: Insights into pathogenesis, diagnostic challenges, and therapeutic options. The American Journal of Medicine 121: 748-757.

50. Bugger H, Abel ED (2014) Molecular mechanisms of diabetic cardiomyopathy. Diabetologia 57: 660-671.

51. Westermeier F (2015) Defective insulin signaling and mitochondrial dynamics in diabetic cardiomyopathy. Biochimica et Biophysica Acta (BBA)-Molecular Cell Research 1853: 1113-1118.

52. Rodrigues B (1998) Metabolic disturbances in diabetic cardiomyopathy. In Cardiac Metabolism in Health and Disease (pp. 53-57). Springer US.

53. Liu Q (2014) Diabetic cardiomyopathy and its mechanisms: Role of oxidative stress and damage. Journal of Diabetes Investigation 5: 623-634.

54. Dobrin JS (2010) Diabetic cardiomyopathy: Signaling defects and therapeutic approaches. Expert Review of Cardiovascular Therapy 8: 373-391.

55. Bugger H (2008) Type 1 diabetic akita mouse hearts are insulin sensitive but manifest structurally abnormal mitochondria that remain coupled despite increased uncoupling protein 3. Diabetes 57: 2924-2932.

56. Buchanan J (2005) Reduced cardiac efficiency and altered substrate metabolism precedes the onset of hyperglycemia and contractile dysfunction in two mouse model of insulin resistance and obesity. Endocrinology 146: 5341-5349.

57. Peterson LR (2004) Effect of obesity and insulin resistance on myocardial substrate metabolism and efficiency in young women. Circulation 109: 2191-2196.

58. Gibbs EM (1995) Glycemic improvement in diabetic db/db mice by overexpression of the human insulin-regulatable glucose transporter (GLUT4). Journal of Clinical Investigation 95: 1512 .

59. Belke DD (2000) Altered metabolism causes cardiac dysfunction in perfused hearts from diabetic $(\mathrm{db} / \mathrm{db})$ mice. American Journal of Physiology-Endocrinology and Metabolism 279: E1104-E1113.

60. Wright JJ (2009) Mechanisms for increased myocardial fatty acid utilization following short-term high-fat feeding. Cardiovascular research 82: 351-360.

61. Malhotra A, Sanghi V (1997) Regulation of contractile proteins in diabetic heart. Cardiovascular Research 34: 34-40.

62. Takeda N (1988) Myocardial mechanical and myosin isoenzyme alterations in streptozotocin-diabetic rats. Japanese Heart Journal 29: 455-463. 
Albakri A (2018) Diabetic cardiomyopathy: a review of literature on clinical status and meta-analysis of tissue doppler diagnostic method and the clinical value of intensive glycemic control

63. Abe T (2002) Left ventricular diastolic dysfunction in type 2 diabetes mellitus model rats. American Journal of Physiology-Heart and Circulatory Physiology 282: H138-H148.

64. Mazumder PK (2004) Impaired cardiac efficiency and increased fatty acid oxidation in insulin-resistant ob/ob mouse hearts. Diabetes 53: 2366-2374.

65. Cai L (2002) Hyperglycemia-induced apoptosis in mouse myocardium. Diabetes 51: 1938-1948.

66. Huynh K (2013) Targeting the upregulation of reactive oxygen species subsequent to hyperglycemia prevents type 1 diabetic cardiomyopathy in mice. Free Radical Biology and Medicine 60: 307-317.

67. Varma A (2012) Anti-inflammatory and cardioprotective effects of tadalafil in diabetic mice. PloS one 7: e45243.

68. Chowdhry F (2007) Diabetes increases apoptosis and necrosis in both ischemic and nonischemic human myocardium: Role of caspases and poly-adenosine diphosphateribose polymerase. The Journal of Thoracic and Cardiovascular Surgery 134: 124-131.

69. Fiordaliso F (2001) Hyperglycemia activates p53 and p53-regulated genes leading to myocyte cell death. Diabetes 50: 2363-2375.

70. Kobayashi S (2015) Autophagy and mitophagy in diabetic cardiomyopathy. Biochimica et Biophysica Acta (BBA)-Molecular Basis of Disease 1852: 252-261.

71. Varga ZV (2015) Interplay of oxidative, nitrosative/nitrative stress, inflammation, cell death and autophagy in diabetic cardiomyopathy. Biochimica et Biophysica Acto (BBA)-Molecular Basis of Disease 1852: 232-242.

72. Chen S (2000) Diabetes-induced myocardial structural changes: role of endothelin-1 and its receptors. Journal of Molecular and Cellular Cardiology 32: 1621-1629.

73. Fischer VW (1984) Pathomorphologic aspects of muscular tissue in diabetes mellitus. Human Pathology 15: 1127-1136.

74. Shimizu M, Umeda K (1993) Collagen remodelling in myocardia of patients with diabetes. Journal of Clinical Pathology 46: 32-36.

75. Anversa P (1998) Myocyte death and growth in the failing heart. Laboratory Investigation; a Journal of Technical Methods and Pathology 78: 767.

76. Zieman SJ (2004) Advanced glycation end-product crosslinking in the cardiovascular system: Potential therapeutic target for cardiovascular disease. Drugs 64: 459-470.

77. Hamby RI (1974) Diabetic Cardiomyopathy. JAMA 229: 1749-1754.

78. Sunni S (1986) Diabetic Cardiomyopathy. A morphologic study of intramyocardial arteries. Archives Pathology Laboratory Medicine 110: 375-381.

79. Zhi YF (2004) Diabetic cardiomyopathy: Evidences, mechanism and therapeutic implications. Endocrine Reviews 25: 543-567.

80. Duncan JG (2011) Mitochondrial dysfunction in diabetic cardiomyopathy. Biochimica et Biophysica Acta (BBA)-Molecular Cell Research 1813: 1351-1359.

81. Anderson EJ (2009) Substrate-specific derangements in mitochondrial metabolism and redox balance in the atrium of the type 2 diabetic human heart. Journal of American College of Cardiology 54: 1891-1898.

82. Anderson EJ (2011) Increased propensity for cell death in diabetic human heart is mediated by mitochondrial-dependent pathways. American Journal of PhysiologyHeart and Circulatory Physiology 300: H118-H12.

83. Ku TH (1985) Oxidative metabolism of Polytron versus Nagarse mitochondria in hearts of genetically diabetic mice. Biochim Biophys Acta 806: 9-15.

84. Bugger H, Abel ED (2009) Rodent models of diabetic cardiomyopathy. Disease Models \& Mechanisms, 2: 454-466.

85. Bugger H, Abel ED (2008) Molecular mechanisms for myocardial mitochondrial dysfunction in the metabolic syndrome. Clinical Science 114: 195-210.

86. Konig A (2012) Diabetes mellitus and myocardial mitochondrial dysfunction: bench to bedside. Heart Failure Clinics 8: 551-561.

87. Maron BJ (2006) Contemporary definitions and classification of the cardiomyopathies. Circulation, 113: 1807-1816.

88. Maya L, Villarreal FJ (2010) Diagnostic approaches for diabetic cardiomyopathy and myocardial fibrosis. Journal of Molecular and Cellular Cardiology 48: 524-529.

89. Zile MR (2001) Heart failure with a normal ejection fraction. Circulation 104: 779-782.

90. Galderisi M (2006) Diastolic dysfunction and diabetic cardiomyopathy: Evaluation by Doppler echocardiography. Journal of the American College of Cardiology 48: 15481551 .
91. Chuang ML (2000) Three-dimensional echocardiographic measurement of left ventricular mass: comparison with magnetic resonance imaging and two-dimensional echocardiographic determinations in man. International Journal of Cardiac Imaging 16: 347-357.

92. Doyle M, Fuisz A (2003) The Impact of Myocardial Flow Reserve on the Detection of Coronary Artery Disease by Perfusion Imaging Methods: An NHLBI WISE Study: PERFUSION IMAGING. Journal of Cardiovascular Magnetic Resonance 5: 475-485.

93. Ficaro EP (2004) Advances in quantitative perfusion SPECT imaging. Journal of Nuclear Cardiology 11: 62-70.

94. Maisel AS (2003) Bedside B-type natriuretic peptide in the emergency diagnosis of heart failure with reduced or preserved ejection fraction. Results from the Breathing Not Properly Multinational Study. Journal of American College of Cardiology 41: 2010-2017.

95. Epshteyn V (2003) Utility of B-type natriuretic peptide (BNP) as a screen for left ventricular dysfunction in patients with diabetes. Diabetes Care 26: 2081-2087.

96. Khan KA (2006) Prevalence of diastolic dysfunction in normotensive asymptomatic patients with well-controlled type 2 diabetes mellitus. Journal of Chinese Clinical Medicine 1: 193-200.

97. Zabalgoitia M (2001) Prevalence of diastolic dysfunction in normotensive, asymptomatic patients with well-controlled type 2 diabetes mellitus. The American Journal of Cardiology 87: 320-323.

98. From AM (2010) The development of heart failure in patients with diabetes mellitus and pre-clinical diastolic dysfunction: A population-based study. Journal of the American College of Cardiology 55: 300-305.

99. Poirier P (2001) Diastolic dysfunction in normotensive men with well-controlled type 2 diabetes. Diabetes Care 24: 5-10.

100. Liu JE (2001) The impact of diabetes on left ventricular filling pattern in normotensive and hypertensive adults: The Strong Heart Study. Journal of the American College of Cardiology 37: 1943-1949.

101. Eren M (2004) Relation between aortic stiffness and left ventricular diastolic function in patients with hypertension, diabetes, or both. Heart 90: 37-43.

102. Zarich SW (1988) Diastolic abnormalities in young asymptomatic diabetic patients assessed by pulsed Doppler echocardiography. Journal of the American College of Cardiology 12: 114-120.

103. Ha JW (2007) Abnormal left ventricular longitudinal functional reserve in patients with diabetes mellitus: implication for detecting subclinical myocardial dysfunction using exercise tissue Doppler echocardiography. Heart 93: 1571-1576.

104. Fang (2003) Echocardiographic detection of early diabetic myocardial disease Journal of the American College of Cardiology 41: 611-617.

105. Andersen NH (2003) Decreased left ventricular longitudinal contraction in normotensive and normoalbuminuric patients with Type II diabetes mellitus: A Doppler tissue tracking and strain rate echocardiography study. Clinical Science 105 : 59-66.

106. Nakai H (2009) Subclinical left ventricular dysfunction in asymptomatic diabetic patients assessed by two-dimensional speckle tracking echocardiography: Correlation with diabetic duration. European Journal of Echocardiography 10: 926-932.

107. Palmieri V (2008) Uncomplicated type 1 diabetes and preclinical left ventricular myocardial dysfunction: insights from echocardiography and exercise cardiac performance evaluation. Diabetes research and clinical practice 79: 262-268.

108. Konduracka E (2007) Diabetes-specific cardiomyopathy in type 1 diabetes mellitus: no evidence for its occurrence in the era of intensive insulin therapy. European Heart Journal 28: 2465-2471.

109. Kodama S (2013) Association between physical activity and risk of all-cause mortality and cardiovascular disease in patients with diabetes. Diabetes Care 36 : 471-479.

110. Stolen TO (2009) Interval training normalizes cardiomyocyte function, diastolic $\mathrm{Ca} 2+$ control, and SR Ca2+ release synchronicity in a mouse model of diabetic cardiomyopathy. Circulation Research 105 527-536.

111. Epp RA (2012) Exercise training prevents the development of cardiac dysfunction in the low-dose streptozotocin diabetic rats fed a high-fat diet. Canadian Journal of Physiology and Pharmacology 91: 80-89.

112. Hordern $H$ (2009). Effects of exercise intervention on myocardial function in type 2 diabetes. Heart. 
Albakri A (2018) Diabetic cardiomyopathy: a review of literature on clinical status and meta-analysis of tissue doppler diagnostic method and the clinical value of intensive glycemic control

113. Tahrani AA (2007) Metformin, heart failure, and lactic acidosis: is metformin absolutely contraindicated? British Medical Journal 335: 508.

114. Xie Z (2011) Improvement of cardiac functions by chronic metformin treatment is associated with enhanced cardiac autophagy in diabetic OVE26 mice. Diabetes 60: $1770-1778$.

115. Eurich DT (2007) Benefits and harms of antidiabetic agents in patients with diabetes and heart failure: systematic review. British Medical Journal 335: 497.

116. Aguilar D (2010) Metformin use and mortality in ambulatory patients with diabetes and heart failure. Circulation: Heart Failure 1: 110.

117. Shindler DM (1996) Diabetes mellitus, a predictor of morbidity and mortality in the Studies of Left Ventricular Dysfunction (SOLVD) Trials and Registry. The American journal of cardiology 77: 1017-1020.

118. Ryden L (2000) Efficacy and safety of high-dose lisinopril in chronic heart failure patients at high cardiovascular risk, including those with diabetes mellitus. Results from the ATLAS trial. European Heart Journal 21: 1967-1978.

119. Komajda M (2010) Factors associated with outcome in heart failure with preserved ejection fraction: findings from the Irbesartan in Heart Failure with Preserved Ejection Fraction Study (I-PRESERVE). Circulation: heart failure, circheartfailure 1: 109.

120. Vermes E (2003) Enalapril reduces the incidence of diabetes in patients with chronic heart failure. Circulation 107: 1291-1296.

121. Dickstein K (2002) OPTIMAAL Steering Committee, \& OPTIMAAL Study Group, Effects of losartan and captopril on mortality and morbidity in high-risk patients after acute myocardial infarction: the OPTIMAAL randomised trial. The Lancet 360: 752760.

122. Haas SJ (2003) Are $\beta$-blockers as efficacious in patients with diabetes mellitus as in patients without diabetes mellitus who have chronic heart failure? A meta-analysis of large-scale clinical trials. American Heart Journal 146: 848-853.

123. De Boer RA (2010) Influence of diabetes mellitus and hyperglycemia on prognosis in patients $\geq 70$ years old with heart failure and effects of nebivolol (data from the Study of Effects of Nebivolol Intervention on Outcomes and Rehospitalization in Seniors with Heart Failure [SENIORS]). The American Journal of Cardiology 106: 78-86.

124. Deedwania PC (2005) Efficacy, safety and tolerability of metoprolol CR/XL in patients with diabetes and chronic heart failure: experiences from MERIT-HF. American Heart Journal 149: 159-167.

125. Investigators CI (1999) The cardiac insufficiency bisoprolol study II (CIBIS-II): a randomised trial. The Lancet 353: 9-13.

126. Packer M (2001) Effect of carvedilol on survival in severe chronic heart failure. New England Journal of Medicine 344: 1651-1658.

127. Poole-Wilson PA (2003) Comparison of carvedilol and metoprolol on clinica outcomes in patients with chronic heart failure in the Carvedilol or Metoprolol European Trial (COMET): randomised controlled trial. The Lancet 362: 7-13.
128. Shorr RI (1997) Antihypertensives and the risk of serious hypoglycemia in older persons using insulin or sulfonylureas. JAMA 278: 40-43.

129. Kostis JB (2005) The association of heart failure with insulin resistance and the development of type 2 diabetes. American Journal of Hypertension 18: 731-737.

130. Zannad F (2011) Eplerenone in patients with systolic heart failure and mild symptoms. New England Journal of Medicine 364: 11-21.

131. Afzal NASIR (1989) Influence of verapamil on some subcellular defects in diabetic cardiomyopathy. American Journal of Physiology-Endocrinology and Metabolism 256: E453-E458.

132. Action to Control Cardiovascular Risk in Diabetes Study Group (2008) Effects of intensive glucose lowering in type 2 diabetes. New England Journal of Medicine 2545-2559.

133. UK Prospective Diabetes Study (UKPDS) Group. (1998). Intensive blood-glucose control with sulphonylureas or insulin compared with conventional treatment and risk of complications in patients with type 2 diabetes (UKPDS 33). The lancet 352 : 837-853.

134. Patel A (2007) ADVANCE Collaborative Group, Effects of a fixed combination of perindopril and indapamide on macrovascular and microvascular outcomes in patients with type 2 diabetes mellitus (the ADVANCE trial): a randomised controlled trial. The Lancet 370: 829-840.

135. Dormandy JA (2005) Secondary prevention of macrovascular events in patients with type 2 diabetes in the PROactive Study (PROspective pioglitAzone Clinical Trial In macroVascular Events): A randomised controlled trial. The Lancet 366: 1279-1289.

136. Duckworth WC (2001) Glucose control and cardiovascular complications: the VA Diabetes Trial. Diabetes Care 24: 942-945.

137. Diabetes Control and Complications Trial Research Group (1993) The effect of intensive treatment of diabetes on the development and progression of long-term complications in insulin-dependent diabetes mellitus. New England Journal of Medicine 329: 977-986.

138. Stettler C (2006) Glycemic control and macrovascular disease in types 1 and 2 diabetes mellitus: meta-analysis of randomized trials. American Heart Journal 152: 27-38.

139. Abraira C (2003) The need for glycemic trials in type 2 diabetes. Clinical Diabetes 21: $107-111$.

140. Shichiri M (2000) Long-term results of the Kumamoto Study on optimal diabetes control in type 2 diabetic patients. Diabetes Care 23: B21.

141. Abraira C (1997) Cardiovascular events and correlates in the Veterans Affair Diabetes Feasibility Trial: Veterans Affairs Cooperative Study on glycemic contro and complications in type II diabetes. Archives of Internal Medicine 157: 181-188.

Copyright: (C2018 Albakri A. This is an open-access article distributed under the terms of the Creative Commons Attribution License, which permits unrestricted use, distribution, and reproduction in any medium, provided the original author and source are credited. 